\title{
Sorafenib plus transarterial chemoembolization for unresectable hepatocellular carcinoma
}

\author{
Diamantis I. Tsilimigras, Timothy M. Pawlik \\ Department of Surgery, Division of Surgical Oncology, The Ohio State University Wexner Medical Center and James Comprehensive Cancer \\ Center, Columbus, OH, USA \\ Correspondence to: Diamantis I. Tsilimigras, MD. Department of Surgery, Division of Surgical Oncology, The Ohio State University, Wexner Medical \\ Center, 410 W 10th Ave, Columbus, OH 43210, USA. Email: diamantis.tsilimigras@osumc.edu. \\ Comment on: Li H, Li S, Geng J, et al. Efficacy evaluation of the combination therapy of sorafenib and transarterial chemoembolization for \\ unresectable HCC: a systematic review and meta-analysis of comparative studies. Ann Transl Med 2020;8:540.
}

Submitted Nov 01, 2020. Accepted for publication Nov 09, 2020.

doi: $10.21037 /$ atm-20-7228

View this article at: http://dx.doi.org/10.21037/atm-20-7228

This issue of the Annals of Translational Medicine features an important study by Li and colleagues titled "Efficacy evaluation of the combination therapy of sorafenib and transarterial chemoembolization for unresectable HCC: a systematic review and meta-analysis of comparative studies." (1). The authors performed a systematic review and summarized the current evidence related to the efficacy of combination therapy [i.e., sorafenib plus transarterial chemoembolization (TACE)] versus TACE monotherapy for unresectable hepatocellular carcinoma (HCC) (1). These data add to the current literature and provide the rationale for further research to evaluate the efficacy of combination therapy among patients with unresectable HCC.

According to the American Association for the Study of Liver Diseases (AASLD) and European Association for the Study of the Liver (EASL) guidelines, TACE is recommended for Barcelona Clinic Liver Cancer (BCLC) stage $\mathrm{B}$ patients [i.e., patients with $2-3$ nodules $>3 \mathrm{~cm},>4$ nodules, preserved liver function, performance status (PS) 0 ], while sorafenib is recommended for individuals with BCLC stage C HCC (i.e., portal invasion, extrahepatic spread, preserved liver function, PS 1-2) (2,3). Several clinical trials have demonstrated a survival benefit with TACE over best supportive treatment among patients with unresectable HCC $(4,5)$. TACE delivers anticancer therapy directly to the tumor feeding arteries and blocks the arterial blood supply of liver tumors. In turn, TACE creates a hypoxic tumor microenvironment, that leads to upregulation of the hypoxia inducible factor- 1 , which facilitates tumor angiogenesis with the release of vascular endothelial growth factor (VEGF) and platelet-derived growth factor (PDGF) $(6,7)$. Of note, an increase in serum VEGF levels after TACE has been considered a possible mechanism responsible for high recurrence rates following TACE $(6,7)$. In turn, there has been an interest in combining antiangiogenic agents with TACE to decrease TACE-induced angiogenesis aiming at decreasing recurrence rates. Sorafenib, a multi-kinase inhibitor, inhibits angiogenesis-a major mechanism of cancer progressionby targeting the VEGR receptor 2 (VEGFR2) and PDGF receptor (PDGFR), while also blocking cell proliferation by targeting the MAPK/ERK signaling pathway (8). As such, the addition of sorafenib has been hypothesized to enhance the efficacy of TACE when compared with TACE monotherapy.

In this context, Li and colleagues analyzed 14 studies published between 2011 to 2017 that compared combination therapy (i.e., sorafenib plus TACE) versus monotherapy with TACE (1). In their analysis, the authors included 4 prospective randomized controlled trials (RCTs) (9-12), while the remaining 10 were non-RCTs.(1) The authors demonstrated that the combination of sorafenib and TACE improved time-to-progression (TTP) (HR $=0.72,95 \%$ CI: $0.59-0.88, \mathrm{P}=0.001)$ and overall survival $(\mathrm{OS})(\mathrm{HR}=0.65$, 95\% CI: $0.54-0.79, \mathrm{P}<0.001)$ compared with TACE alone. Several points should be considered when interpreting the results of this meta-analysis (1).

First, half of the included studies were retrospective in 
nature which was problematic, and may have introduced selection bias (1). Of note, there was large heterogeneity in the characteristics of patients included in each study. In particular, although the majority of studies analyzed BCLC-B and C stage patients, two studies included BCLC-A patients who-according to the latest BCLC guidelines-should be recommended resection, transplantation or ablation (2). In addition, three out of the 4 analyzed RCTs had negative results and did not demonstrate a significant benefit of combination therapy over TACE monotherapy (9-12). In turn, the survival benefit associated with combination therapy in the cumulative analysis was presumably mainly driven by the results of the non-RCTs. Although the results of the non-RCTs should not be ignored, the heterogeneity of these cohorts, as well as the chance of selection bias in retrospective studies, limit the significance of these findings. A subgroup analysis among RCTs or prospective studies (RCT and non-RCTs) could have provided a higher-level of evidence regarding the efficacy of combination therapy and could have enhanced the validity of the current metaanalysis (1).

Li et al. analyzed studies that reported on TACE in the form of both conventional TACE (cTACE) and drug eluting beads (DEB)-TACE (1). As previously demonstrated, DEBTACE may enhance drug delivery, reduce systemic drug exposure and eventually reduce adverse events compared with cTACE $(13,14)$. The authors reported that cTACE and DEB-TACE revealed comparable results relative to TTP $(\mathrm{P}=0.15)$, yet a subgroup analysis comparing combination therapy versus TACE monotherapy stratified by the type of TACE would have been informative. Performing such a subgroup analysis based on the type of TACE (i.e., cTACE or DEB-TACE) may provide more insight into which TACE approach provided the most benefit when combined with sorafenib.

In addition, the majority of the studies were conducted in Asian countries (10 out of 14) (1), where the etiology of HCC is somewhat different compared with Western countries. Indeed, hepatitis B virus (HBV) infection is the main predisposing factor for the development of HCC in Asia, while non-alcoholic fatty liver disease (NAFLD), alcohol-related cirrhosis and hepatitis $\mathrm{C}$ virus (HCV) infection are the main attributable factors for HCC in Western countries $(2,3)$. Previous investigators have suggested that sorafenib may have a differential effect on outcomes according to the etiology of HCC (15). Indeed, a recent meta-analysis of randomized phase III trials demonstrated a sorafenib benefit only among HCVpositive and $\mathrm{HBV}$-negative patients as opposed to any other combination of viral status (16). Of note, the relative impact of sorafenib across all other etiologies of HCC (i.e., NAFLD, alcohol-related cirrhosis etc.) is not well understood to date and warrants further investigation. As such, results from the present meta-analysis need to be interpreted in light of the population analyzed and not unequivocally extrapolated to other populations with different characteristics (i.e., Western populations).

Another factor that should be considered was the inclusion of studies that were published only up to December 2017. Since then, a number of studies have been published on the topic, yet the majority compared combination therapy versus sorafenib monotherapy and not TACE monotherapy (which was the objective of the present meta-analysis) (17-19). Of note, an important multicenter prospective RCT from Japan (TACTICS trial) was recently published that evaluated the combination of TACE plus sorafenib versus TACE alone (20). This trial was performed by the same group as the first trial by Kudo et al. that was published in 2011 (9), yet the TACTICS trial was a collaborative effort from multiple Japanese centers (20). The authors utilized a new protocol compared with the first trial that had yielded negative results (9). Specifically, patients in the combination group received sorafenib for 2-3 weeks prior to TACE, which was continued during ondemand cTACE sessions; in contrast, in the previous trial, $50 \%$ of patients had sorafenib $>9$ weeks following TACE. Furthermore, in the TACTICS trial, the investigators administered a higher dose of sorafenib (400 $\mathrm{mg}$ once daily for $2-3$ weeks before TACE followed by $800 \mathrm{mg}$ once daily during cTACE) versus the initial trial $(400 \mathrm{mg})$ for a longer period of time (median time of sorafenib treatment: $38.7 \mathrm{vs}$. 17.1 weeks). Adverse events were consistent with previous TACE combination trials (21). The authors concluded that TACE plus sorafenib significantly improved progressionfree survival (PFS) and TTP over TACE monotherapy among patients with unresectable HCC (20). As such, optimizing combination therapy protocols (i.e., timing of sorafenib administration, duration and dosage) may impact TTP and OS following combination therapy.

More recently, a number of other novel antiangiogenic agents have been investigated to treat advanced HCC. In the phase III REFLECT trial, lenvatinib demonstrated improved PFS and an objective response rate with equivalent OS compared with sorafenib in the setting of unresectable HCC (22). In addition, the phase III 
IMbrave150 trial demonstrated that the combination of atezolizumab-bevacizumab resulted in better OS and PFS compared with sorafenib alone among patients with unresectable HCC (23). As such, newer agents such as lenvatinib and/or atezolizumab-bevacizumab may be even more potent anti-VEGF agents compared with sorafenib. In turn, these agents may more efficiently counteract the upregulation of hypoxia-induced angiogenesis caused by TACE. Although these novel treatments have yet to be approved as standard of care for individuals with advanced HCC, the combination of TACE along with these novel anti-angiogenic agents represents a promising field for future research.

In conclusion, the mechanistic background of TACEinduced angiogenesis provides a rationale for the implementation of anti-angiogenic agents along with TACE to reduce recurrence. The findings by $\mathrm{Li}$ et al. add to the current literature and suggest that the combination of TACE with sorafenib may provide a benefit over TACE alone for patients with unresectable HCC. The present meta-analysis provides a strong argument for the implementation of further large scale, prospective RCTs to validate the timing and the optimal dosage of sorafenibor other more novel agents-to provide better outcomes when combined with TACE, while also evaluating adverse events and drug-related toxicity. The use of novel antiangiogenic agents with TACE is a promising field for future research.

\section{Acknowledgments}

Funding: None.

\section{Footnote}

Provenance and Peer Review: This article was commissioned by the editorial office, Annals of Translational Medicine. The article did not undergo external peer review.

Conflicts of Interest: Both authors have completed the ICMJE uniform disclosure form (available at http://dx.doi. org/10.21037/atm-20-7228). Both authors have no conflicts of interests to declare.

Ethical Statement: The authors are accountable for all aspects of the work in ensuring that questions related to the accuracy or integrity of any part of the work are appropriately investigated and resolved.
Open Access Statement: This is an Open Access article distributed in accordance with the Creative Commons Attribution-NonCommercial-NoDerivs 4.0 International License (CC BY-NC-ND 4.0), which permits the noncommercial replication and distribution of the article with the strict proviso that no changes or edits are made and the original work is properly cited (including links to both the formal publication through the relevant DOI and the license). See: https://creativecommons.org/licenses/by-nc-nd/4.0/.

\section{References}

1. Li H, Li S, Geng J, et al. Efficacy evaluation of the combination therapy of sorafenib and transarterial chemoembolization for unresectable HCC: a systematic review and meta-analysis of comparative studies. Ann Transl Med 2020;8:540.

2. European Association for the Study of the Liver. EASL Clinical Practice Guidelines: Management of hepatocellular carcinoma. J Hepatol 2018;69:182-236.

3. Marrero JA, Kulik LM, Sirlin CB, et al. Diagnosis, Staging, and Management of Hepatocellular Carcinoma: 2018 Practice Guidance by the American Association for the Study of Liver Diseases. Hepatology 2018;68:723-50.

4. Llovet JM, Real MI, Montana X, et al. Arterial embolisation or chemoembolisation versus symptomatic treatment in patients with unresectable hepatocellular carcinoma: a randomised controlled trial. Lancet 2002;359:1734-9.

5. Llovet JM, Bruix J. Systematic review of randomized trials for unresectable hepatocellular carcinoma: Chemoembolization improves survival. Hepatology 2003;37:429-42.

6. Wang B, Xu H, Gao ZQ, et al. Increased expression of vascular endothelial growth factor in hepatocellular carcinoma after transcatheter arterial chemoembolization. Acta Radiol 2008;49:523-9.

7. Li X, Feng GS, Zheng CS, et al. Expression of plasma vascular endothelial growth factor in patients with hepatocellular carcinoma and effect of transcatheter arterial chemoembolization therapy on plasma vascular endothelial growth factor level. World J Gastroenterol 2004;10:2878-82.

8. Llovet JM, Ricci S, Mazzaferro V, et al. Sorafenib in advanced hepatocellular carcinoma. $\mathrm{N}$ Engl J Med 2008;359:378-90.

9. Kudo M, Imanaka K, Chida N, et al. Phase III study of sorafenib after transarterial chemoembolisation in Japanese 
and Korean patients with unresectable hepatocellular carcinoma. Eur J Cancer 2011;47:2117-27.

10. Sansonno D, Lauletta G, Russi S, et al. Transarterial chemoembolization plus sorafenib: a sequential therapeutic scheme for HCV-related intermediate-stage hepatocellular carcinoma: a randomized clinical trial. Oncologist 2012;17:359-66.

11. Hoffmann K, Ganten T, Gotthardtp D, et al. Impact of neo-adjuvant Sorafenib treatment on liver transplantation in HCC patients - a prospective, randomized, doubleblind, phase III trial. BMC Cancer 2015;15:392.

12. Lencioni R, Llovet JM, Han G, et al. Sorafenib or placebo plus TACE with doxorubicin-eluting beads for intermediate stage HCC: The SPACE trial. J Hepatol 2016;64:1090-8.

13. Lammer J, Malagari K, Vogl T, et al. Prospective randomized study of doxorubicin-eluting-bead embolization in the treatment of hepatocellular carcinoma: results of the PRECISION V study. Cardiovasc Intervent Radiol 2010;33:41-52.

14. Varela M, Real MI, Burrel M, et al. Chemoembolization of hepatocellular carcinoma with drug eluting beads: efficacy and doxorubicin pharmacokinetics. J Hepatol 2007;46:474-81.

15. Bruix J, Cheng AL, Meinhardt G, et al. Prognostic factors and predictors of sorafenib benefit in patients with hepatocellular carcinoma: Analysis of two phase III studies. J Hepatol 2017;67:999-1008.

16. Jackson R, Psarelli EE, Berhane S, et al. Impact of Viral Status on Survival in Patients Receiving Sorafenib for Advanced Hepatocellular Cancer: A Meta-Analysis of Randomized Phase III Trials. J Clin Oncol 2017;35:622-8.

Cite this article as: Tsilimigras DI, Pawlik TM. Sorafenib plus transarterial chemoembolization for unresectable hepatocellular carcinoma. Ann Transl Med 2020;8(23):1557. doi: 10.21037/atm$20-7228$
17. Lee SW, Lee TY, Peng YC, et al. The therapeutic benefits of combined sorafenib and transarterial chemoembolization for advanced hepatocellular carcinoma. J Dig Dis 2020;21:287-92.

18. Kimura Y, Kaneko R, Yano Y, et al. The Prognosis of Hepatocellular Carcinoma Treated with Sorafenib in Combination with TACE. Asian Pac J Cancer Prev 2020;21:1797-805.

19. Chien SC, Chen CY, Cheng PN, et al. Combined Transarterial Embolization/Chemoembolization-Based Locoregional Treatment with Sorafenib Prolongs the Survival in Patients with Advanced Hepatocellular Carcinoma and Preserved Liver Function: A Propensity Score Matching Study. Liver Cancer 2019;8:186-202.

20. Kudo M, Ueshima K, Ikeda M, et al. Randomised, multicentre prospective trial of transarterial chemoembolisation (TACE) plus sorafenib as compared with TACE alone in patients with hepatocellular carcinoma: TACTICS trial. Gut 2020;69:1492-501.

21. Pawlik TM, Reyes DK, Cosgrove D, et al. Phase II trial of sorafenib combined with concurrent transarterial chemoembolization with drug-eluting beads for hepatocellular carcinoma. J Clin Oncol 2011;29:3960-7.

22. Yamashita T, Kudo M, Ikeda K, et al. REFLECT-a phase 3 trial comparing efficacy and safety of lenvatinib to sorafenib for the treatment of unresectable hepatocellular carcinoma: an analysis of Japanese subset. J Gastroenterol 2020;55:113-22.

23. Finn RS, Qin S, Ikeda M, et al. Atezolizumab plus Bevacizumab in Unresectable Hepatocellular Carcinoma. N Engl J Med 2020;382:1894-905. 